\title{
Intrinsic surface dipole in topological insulators
}

\author{
Benjamin M. Fregoso ${ }^{1}$ and Sinisa $\mathrm{Coh}^{1,2}$ \\ ${ }^{1}$ Department of Physics, University of California, Berkeley, California, 94720, USA \\ ${ }^{2}$ Materials Sciences Division, Lawrence Berkeley National Laboratory, Berkeley, California 94720, USA
}

We calculate the local density of states of two prototypical topological insulators $\left(\mathrm{Bi}_{2} \mathrm{Se}_{3}\right.$ and $\mathrm{Bi}_{2} \mathrm{Te}_{2} \mathrm{Se}$ ) as a function of distance from the surface within density functional theory. We find that, in the absence of disorder or doping, there is a $2 \mathrm{~nm}$ thick surface dipole the origin of which is the occupation of the topological surface states above the Dirac point. As a consequence, the bottom of the conduction band is bent upward by about $75 \mathrm{meV}$ near the surface, and there is a hump-like feature associated with the top of the valence band. We expect that band bending will occur in all pristine topological insulators as long as the Fermi level does not cross the Dirac point. Our results show that topological insulators are intrinsic Schottky barrier solar cells.

PACS numbers: 73.20.At,73.30.+y,71.20.-b,77.90.+k,73.50.-h, 73.61.-r

\section{INTRODUCTION}

Semiconductor interfaces are of fundamental importance in electronic device operation. At the heart of this technology are heterojunctions made of n-doped and p-doped semiconductors ${ }^{1}$. The charge carriers through $p n$-junctions can be accurately controlled via gate voltages, giving diodes and transistors its useful properties. It is well known that near the interface region there is a permanent electric field, which bends the conduction and valence levels. This region is referred to as spacecharge region or interface dipole (surface dipole if the interface is with vacuum). This band-bending (BB) effect is also of fundamental importance in silicon-based solar cell technology, where the electric field separates photo-exited electrons and holes.

Band bending in semiconductors can be determined from the I-V characteristics of the junction, from surface photo-voltage spectroscopy ${ }^{2}$, surface potential microscopy or from Angle-Resolved Photoemission Spectroscopy (ARPES). Many factors contribute to the BB measured by any of these techniques. Among the most important are the difference in work functions of the materials at the interface, the image charge potential and possible interface reconstruction. These are 'intrinsic' contributions. Occupied impurity states also contribute to BB (extrinsic contributions). Analytical calculations of $\mathrm{BB}$ in semiconductor interfaces usually determine the charge distribution self-consistently from an effective one-dimensional Poisson equation and a singleparticle Schrodinger equation. Such calculations often depend on unknown parameters, which are ultimately determined in experiments.

Recently, a new type of 'semiconductor' was discovered, the topological insulator (TI), which is an insulator in the bulk but has metallic surface states (SSs) protected by time reversal symmetry ${ }^{3-7}$. For further details about TIs we refer the reader to the many excellent reviews of the subject such as Ref. 8 and references therein. At low energies, the surface states of TIs can be described by the Dirac equation in two-dimensions with the spin of

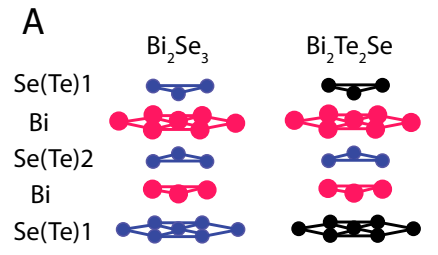

B SSs occupied up to DP

C

\begin{tabular}{lcc}
\multicolumn{1}{c}{ Quantity } & $\mathrm{Bi}_{2} \mathrm{Se}_{3}$ & $\mathrm{Bi}_{2} \mathrm{Te}_{2} \mathrm{Se}$ \\
\hline \hline Slab thickness $(\mathrm{nm})$ & 8.4 & 8.9 \\
\hline Bulk gap (meV) & 350 & 250 \\
\hline Surface state width $(\mathrm{nm})$ & $\sim 1$ & $\sim 1$ \\
\hline Conduction BB $\left(\mathrm{E}_{1}\right)(\mathrm{meV})$ & 75 & 60 \\
\hline Valence BB $\left(\mathrm{E}_{2}\right)$ & 20 & 20 \\
\hline Hump-like feature $\left(\mathrm{E}_{3}\right)$ & 65 & 30
\end{tabular}

D

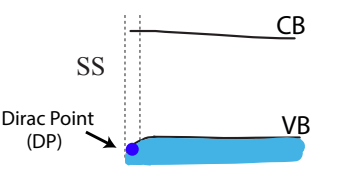
Physical case

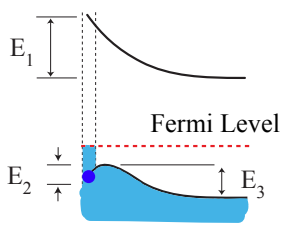

FIG. 1. (Color online) (A) Hexagonal crystal structure of $\mathrm{Bi}_{2} \mathrm{Se}_{3}$ and $\mathrm{Bi}_{2} \mathrm{Te}_{2} \mathrm{Se}$ quintuple layers. (B) schematic diagram shows a cross section of the slab and the location of the valence band (VB) and conduction band (CB). Occupied regions are shaded. Two cases are shown: (B) when the surface states (SSs) are occupied up to the Dirac point (DP) and (D) when they are thermally occupied. When the SSs are occupied up the the DP only (non-thermal case), upward band bending (BB) is absent but there is a small downward BB near the surface. When SSs are occupied (thermal case), the bands bend upward near the surface of the slab. In (C) we show the $\mathrm{BB}$ characteristics of $\mathrm{Bi}_{2} \mathrm{Se}_{3}$ and $\mathrm{Bi}_{2} \mathrm{Te}_{2} \mathrm{Se} . \mathrm{E}_{1,2,3}$ parametrize the magnitude of $\mathrm{BB}$.

the electron locked to its momentum. This property can be utilized in spintronic ${ }^{9}$ applications.

Band bending in TIs surfaces has been investigated experimentally with ARPES ${ }^{10-17}$ and other techniques ${ }^{18,19}$. However, the results have not been consistent, even in apparently similar conditions. For example, $\mathrm{BB}$ of $\mathrm{Bi}_{2} \mathrm{Se}_{3}$ has been found to be either upward $(\sim 75 \mathrm{meV})^{12}$ or downward $(\sim 100-300 \mathrm{meV})^{10,11,13,16,17,20}$. This suggest that the dominant contribution to $\mathrm{BB}$ in these experiments is extrinsic, e.g., doping, and hence it is very sensitive to the details of the sample preparation. In view of these considerations, it is clear that extrinsic and intrin- 
sic effects play a role in actual experiments and that disentangling intrinsic from extrinsic factors would be very useful as a benchmark for future experimental and theoretical studies. On the theoretical side, the PoissonSchrodinger equations ${ }^{21,22}$ and tight-binding models ${ }^{23,24}$ have been successfully used to model BB in TIs. However, understanding and characterizing BB in TIs interfaces has not been explored systematically from first principles despite the fact that it could open the door for new technological applications, e.g., in solar cell technology.

In this paper we resolve the intrinsic contribution to $\mathrm{BB}$ (and hence to the surface dipole) by studying $\mathrm{BB}$ in pristine TIs $\mathrm{Bi}_{2} \mathrm{Se}_{3}$ and $\mathrm{Bi}_{2} \mathrm{Te}_{2} \mathrm{Se}$. We consider a finite slab of $\mathrm{Bi}_{2} \mathrm{Se}_{3}$ and $\mathrm{Bi}_{2} \mathrm{Te}_{2} \mathrm{Se}$ and calculate variations of the local density of states (LDOS) as a function of depth using first principles density functional theory (DFT) with no fitting parameters. Fig. 1 summarizes our main results. We find an upward BB of the conduction and valence bands within $2 \mathrm{~nm}$ below the surface. The upward $\mathrm{BB}$ for $\mathrm{Bi}_{2} \mathrm{Se}_{3}$ and $\mathrm{Bi}_{2} \mathrm{Te}_{2} \mathrm{Se}$ is $75 \mathrm{meV}$ and $60 \mathrm{meV}$ respectively, even without disorder or doping. We attribute this $\mathrm{BB}$ to the occupation of topological SSs above the Dirac point. In the valence band we find a hump-like feature near the surface consisting of an upward bend ( $65 \mathrm{meV}$ for $\mathrm{Bi}_{2} \mathrm{Se}_{3}$ and $30 \mathrm{meV}$ for $\mathrm{Bi}_{2} \mathrm{Te}_{2} \mathrm{Se}$ ) $2 \mathrm{~nm}$ from the surface, followed by a $20 \mathrm{meV}$ downward bend $1 \mathrm{~nm}$ from the surface. We note that, our results do not rely on the Poisson equation to find the equilibrium charge distribution. Instead, the charge distribution arises from the inclusion of electron correlations at the DFT level. Therefore, we expect deviations from Poisson electrostatics. In particular, we find asymmetric $\mathrm{BB}$ of the valence and conduction bands, including a small downward $\mathrm{BB}$ of the valence band near the surface. Finally, although we used $\mathrm{Bi}_{2} \mathrm{Se}_{3}$ and $\mathrm{Bi}_{2} \mathrm{Te}_{2}$ Se as TI prototypes, we expect similar order of magnitude $\mathrm{BB}$ for the family $\mathrm{Bi}_{2} \mathrm{X}_{3}$ with $\mathrm{X}=\mathrm{Se}, \mathrm{Te}$ and $\mathrm{X}_{2} \mathrm{Te}_{2} \mathrm{Y}$ with $\mathrm{X}=\mathrm{Sb}, \mathrm{Bi}$ and $\mathrm{Y}=\mathrm{S}, \mathrm{Se}$, as $\mathrm{Y}$ and $\mathrm{X}$ enter with the same oxidation state.

This paper is organized as follows. In Sec. II we compute the band structure and LDOS of $\mathrm{Bi}_{2} \mathrm{Se}_{3}$ and $\mathrm{Bi}_{2} \mathrm{Te}_{2}$ Se for the physically relevant situation where thermalized electrons fill the SSs above the Dirac point. In Sec. III we compare with the LDOS without spin-orbit coupling and with SSs occupied up to the DP and discuss the origin of the surface dipole. The appendix A contains additional information about the calculation.

\section{LOCAL DENSITY OF STATES}

\section{A. Crystal structure}

$\mathrm{Bi}_{2} \mathrm{Se}_{3}$ and $\mathrm{Bi}_{2} \mathrm{Te}_{2}$ Se are layered materials with rhombohedral $(\mathrm{R} \overline{3} \mathrm{~m})$ crystal structure ${ }^{25}$. The structure can be represented as hexagonal planes stacked along the $c$-axis containing only one type of atom, as shown in Fig. 1A. In one hexagonal unit cell, the order of
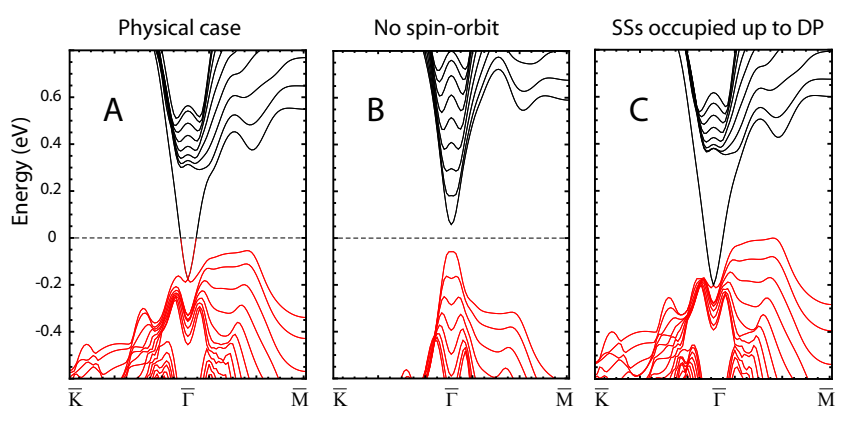

FIG. 2. (Color online) Band structure of a slab of $\mathrm{Bi}_{2} \mathrm{Se}_{3}$. (A) corresponds to the physical case where electrons are thermalized at $300 \mathrm{~K}$ and occupy surface states (SSs) above the Dirac point (DP) which is located at the $\bar{\Gamma}$ point. In (B) the spinorbit coupling has been turned off and hence there are no SSs. In (C) the SSs are occupied up to the DP. In all panels occupied bands are marked in red.

the stacking is -[Se(2)-Bi-Se(1)-Bi-Se(2) $]_{0}-[\mathrm{Se}(2)-\mathrm{Bi}-\mathrm{Se}(1)-$ $\mathrm{Bi}-\mathrm{Se}(2)]_{1 / 3}-[\mathrm{Se}(2)-\mathrm{Bi}-\mathrm{Se}(1)-\mathrm{Bi}-\mathrm{Se}(2)]_{2 / 3}$ in $\mathrm{Bi}_{2} \mathrm{Se}_{3}$ and $[\mathrm{Te}-\mathrm{Bi}-\mathrm{Se}-\mathrm{Bi}-\mathrm{Te}]_{0}-[\mathrm{Te}-\mathrm{Bi}-\mathrm{Se}-\mathrm{Bi}-\mathrm{Te}]_{1 / 3^{-}}[\mathrm{Te}-\mathrm{Bi}-\mathrm{Se}-\mathrm{Bi}-\mathrm{Te}]_{2 / 3^{-}}$in $\mathrm{Bi}_{2} \mathrm{Te}_{2} \mathrm{Se}$. The group of five atomic layers in square brackets are often referred to as a quintuple layer (QL) shown in Fig. 1A. The subscripts indicate the fractional translation of the QL along the $c$-axis of the hexagonal unit cell. The QLs are weakly coupled by Van der Waals forces, forming natural cleavage planes with negligible surface reconstruction. In what follows we present results for $\mathrm{Bi}_{2} \mathrm{Se}_{3}$ only, the results for $\mathrm{Bi}_{2} \mathrm{Te}_{2} \mathrm{Se}$ are similar and summarized in Fig. 1.

\section{B. Band structure and local density of states}

Here we provide some details of our first-principles calculation of the LDOS (more information is in the appendix A). We used Quantum-ESPRESSO ${ }^{26}$ and Wannier90 ${ }^{27}$ to compute the band structures and LDOS of $\mathrm{Bi}_{2} \mathrm{Se}_{3}$. Our periodic computational unit cell consists of 9 QLs of $\mathrm{Bi}_{2} \mathrm{Se}_{3}$ (total width of $8.4 \mathrm{~nm}$ ) with a $1 \mathrm{~nm}$ of vacuum layer between periodic images. Therefore, each computational cell consists of 45 atoms in total, at which the LDOS is calculated. $\mathrm{Bi}_{2} \mathrm{Se}_{3}$ slabs are terminated with Se atoms on both sides, as found in experiments ${ }^{25}$. Other terminations are possible but generally lead to reconstructed surfaces which are absent in our case.

To visualize $\mathrm{BB}$ we compute the LDOS across the entire slab and then note its variations in intensity as a function of depth. To obtain the LDOS with a required energy resolution we use the Wannier interpolation ${ }^{28}$ method. This method consists of first computing KohnSham orbitals $\psi_{n \mathbf{k}}$ and energies $\epsilon_{n \mathbf{k}}$ on a coarsely sampled Brillouin zone (we used a uniform $9 \times 9$ mesh) for electronic bands of interest. In Fig. 2A we show the band structure of $\mathrm{Bi}_{2} \mathrm{Se}_{3}$. The Dirac point (DP) is at the $\bar{\Gamma}$ point of the Brillouin zone $180 \mathrm{meV}$ below the Fermi 


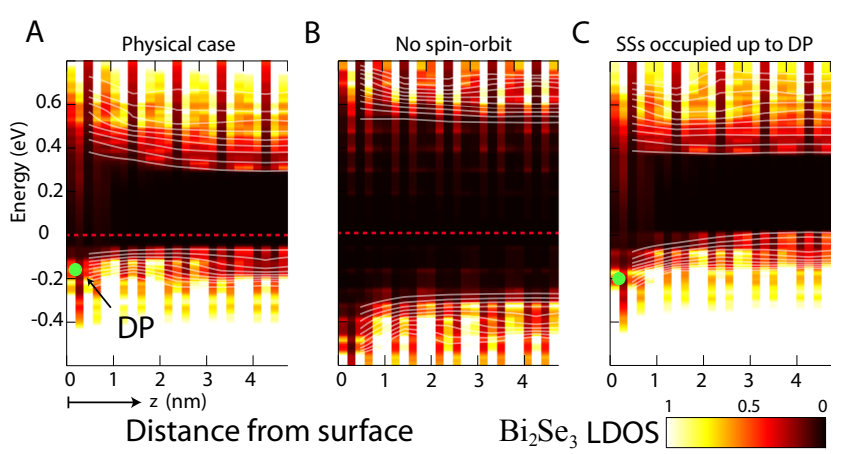

FIG. 3. (Color online) (A) Local density of states (LDOS) across the slab corresponding to the physical case in which electrons are thermalized at $300 \mathrm{~K}$ and occupy SSs above the Dirac point (DP). (B) LDOS with no spin-orbit interaction and (C) occupying surface states (SSs) up to the DP. About half of the slab is shown in each case. Dashed lines indicate the Fermi level when appropriate, and the green dot indicates the position of the DP. The color scale used in panels indicates the LDOS in arbitrary units (largest LDOS corresponds to white color). The energy resolution is $3 \mathrm{meV}$. The white lines are contours of equal intensity of LDOS. The contours where smoothed by Gaussian averaging $(\sigma=36 \mathrm{meV})$ over energy and in each quintuple layer (QL).

level. Note that the SSs (above the DP) are occupied. and there is a bulk gap of $\sim 350 \mathrm{meV}$ consistent with previous reports ${ }^{29-32}$. We then use these orbitals to construct localized Wannier functions ${ }^{33}|\mathbf{R} m i\rangle$ centered at lattice vector $\mathbf{R}$, with both $m=s, p$ like atomic character for all 45 layers in the slab (index $i$ goes from 1 to 45). Since the Hamiltonian is exponentially localized in the Wannier basis we can use this basis to interpolate electronic orbitals and energies on a very dense k-mesh $(3000 \times 3000)$ with a negligible additional computational cost. Finally, we obtain the LDOS from the histogram of the interpolated electronic energies $\epsilon_{n \mathbf{k}}$,

$$
N(E, i)=\sum_{n \mathbf{k} m}\left|\left\langle\mathbf{0} m i \mid \psi_{n \mathbf{k}}\right\rangle\right|^{2} \delta\left(E-\epsilon_{n \mathbf{k}}\right) .
$$

Here the sum is calculated over all electronic states (band $n$ and momentum $\mathbf{k}$ ), and over all Wannier functions $(m=s, p)$, so that the local density of states $N(E, i)$ depends only on atomic layer $(i)$ and energy $(E)$. The LDOS (shown in Fig. 3A) was projected at each of the 45 atomic layers of our supercell. The horizontal axes indicates the approximate distance from the left surface. The matrix element appearing in the sum is independent of lattice vector $\mathbf{R}$, since the Bloch functions are cellperiodic. Therefore we can sum only over $\mathbf{R}=\mathbf{0}$ for simplicity.

Fig. 3A shows the LDOS across a slab of $\mathrm{Bi}_{2} \mathrm{Se}_{3}$ in the physically relevant case with thermalized electrons occupying the SSs. For comparison we also include the case without spin-orbit coupling (B) and with occupied SSs up to the DP only in (C). In this section we discuss the physically relevant case Fig. 3A. The discussion of cases without spin orbit coupling and with partial filling of SSs is given in Sec. III A and Sec. IIIB.

Near the surface there is a non-zero spectral weight extending roughly $d \sim 1 \mathrm{~nm}$ into the bulk, which originates from the topological SSs. Interestingly, one can follow the edges of the valence and conduction bands by observing how the spectral weight intensity varies across the slab. To quantify $\mathrm{BB}$ we follow the following procedure. We define BB by the maximal deviation of the LDOS isocontours (see white lines in Fig. 3A) between the first and middle QLs in a given energy window $(200 \mathrm{meV}$ below the valence band maximum or above the conduction band minimum). With this definition, the edge of the conduction band bends upward by $\sim 75 \mathrm{meV}$ within $2 \mathrm{~nm}$ of the surface, Fig. 3A. On the other hand, the valence band has a hump-like feature near the surface consisting of a $65 \mathrm{meV}$ upward bend $2 \mathrm{~nm}$ from the surface followed by a $20 \mathrm{meV}$ downward bend $1 \mathrm{~nm}$ from the surface.

\section{SURFACE DIPOLE}

In this Section we study the origin of $\mathrm{BB}$ in $\mathrm{Bi}_{2} \mathrm{Se}_{3}$ and show that it can be modeled as a macroscopic surface dipole $^{1}$. We will study both the effect of the spin-orbit interaction and the population of the surface states.

\section{A. Spin-obit interaction}

Turning off the spin-orbit interaction in our calculation reduces the TI to a non-topological insulator, with no topological SSs. This can be seen by the lack of edge states in Fig. 2B. We expect that with no topological SS, the charge distribution across the slab will be affected. This is indeed what we find, see Fig. 3B. Without spinorbit interaction, the conduction band is nearly flat. The maximum bending is less than $20 \mathrm{meV}$. Therefore, we can assign the origin of the upward band bending of the conduction band to the presence of the topological SSs. We focus on the occupation of these states in more detail in the next subsection.

Focusing now on the valence band, we find that even without spin-orbit coupling there is a $75 \mathrm{meV}$ downward BB and a much smaller hump-like feature. Therefore, we again assign the origin of the upward BB to the presence of topological SSs.

\section{B. Population of the surface states}

While the presence of topological SSs is essential for BB in pristine TI slabs, here we discuss the importance of their filling. As discussed in Sec. II, the DP of the $\mathrm{Bi}_{2} \mathrm{Se}_{3}$ slab is below the Fermi level, and hence some of the SSs which are above the DP are populated, Fig. 2A. Since the whole slab is neutral, this excess charge on the 
surface must be compensated by a partial depopulation of some of the bulk-like states.

Fig. 3C shows the LDOS for a $\mathrm{Bi}_{2} \mathrm{Se}_{3}$ slab with SSs occupied up to the Dirac point only and unoccupied above it, see also Fig. 2C. This can be accomplished by setting the population of the first 252 valence bands at all $\mathrm{k}$ points to unity. As can be seen, most of the upward BB features disappear. The conduction $\mathrm{BB}$ is only $20 \mathrm{meV}$ across the slab. For the valence band, maximal upward bending (within $200 \mathrm{meV}$ of the valence band maximum) is only $15 \mathrm{meV}$. However, a downward bend of the valence band near the surface is still present. The downward bend is $90 \mathrm{meV}$, even larger than in the thermalized case.

Therefore, to conclude this and the previous subsection, we find that upward bending of the conduction and valence bands is present only when topological SSs are present (Sec. II A) and when they are occupied.

\section{Magnitude of the surface dipole}

As a consistency check we compare the surface charge density estimated in two ways. First, we calculate the phase space occupied by the SSs from the DP up to the Fermi level in our thermalized calculation. This estimate gives us a surface charge density $n_{2 \mathrm{D}} \sim 0.7 \times 10^{13} \mathrm{~cm}^{-2}$. Second, we estimate the surface charge density by modeling the region near the surface as an electric double layer separated by about $L=2 \mathrm{~nm}$ with opposite charges. One layer is negative due to the occupation of the SS and the other is positive from the depletion of electrons in the bulk-like region. In Fig. 3b, we see that the potential discontinuity at the surface is $\Delta V \sim 75 \mathrm{meV}$ and from $\Delta V=D_{\mathrm{SS}} / \epsilon$ (with $\epsilon \sim 100 \epsilon_{0}$ the permittivity of $\mathrm{Bi}_{2} \mathrm{Se}_{3}{ }^{34}$ ) we obtain $D_{\mathrm{SS}} \sim 6 \times 10^{-11} \mathrm{C} / \mathrm{m}$. Using $D_{\mathrm{SS}}=$ $e n_{2 \mathrm{D}} L$ we find a surface density $n_{2 \mathrm{D}} \sim 2 \times 10^{13} \mathrm{~cm}^{-2}$ in a reasonable agreement with the first estimate, thus confirming that the occupation of SS above the DP is responsible for the observed band bending.

\section{CONCLUSION}

We have shown that free surfaces of pristine $\mathrm{Bi}_{2} \mathrm{Se}_{3}$ and $\mathrm{Bi}_{2} \mathrm{Te}_{2}$ Se with no doping or disorder have an intrinsic surface dipole. $\mathrm{Bi}_{2} \mathrm{Se}_{3}$ and $\mathrm{Bi}_{2} \mathrm{Te}_{2}$ Se grown without any post-processing tend to be doped and disordered and appear to be good bulk conductors ${ }^{11,14,22,35-38}$. However, even in this case the upward contribution to band bending computed in this work is present. We note that Ref. ${ }^{12}$ finds $\sim 75 \mathrm{meV}$ upward band bending in $\mathrm{Bi}_{2} \mathrm{Se}_{3}$ which is similar to pristine $\mathrm{Bi}_{2} \mathrm{Se}_{3}$. However, more analysis is required to attribute this band bending solely to intrinsic effects. As samples become cleaner ${ }^{39}$ only the intrinsic component computed here will remain. We com- ment that, we have considered the case where the DP lies below the Fermi level. We leave for future work the discussion of the case where the Fermi level crosses at or below the DP.

Importantly, the intrinsic band bending found in this work means that TIs generate up to $\sim 75 \mathrm{meV}$ of surface photvoltage ${ }^{2}$ upon illumination. The same principle applies in the operation of Schottky barrier solar cells, where the interface dipole is created at a metalsemiconductor interface. Therefore, TIs could operate as intrinsic Schottky barrier solar cells with an estimated maximum efficiency of $\sim 7 \%$ according to the ShockleyQueisser ${ }^{1}$ criterion.

After completion of this work we became aware of Ref. 40 which considers $\mathrm{BB}$ in $\mathrm{Bi}_{2} \mathrm{Se}_{3}$.

\section{ACKNOWLEDGMENTS}

We thank J. Moore, J. Analytis, M. L. Cohen, C. Salazar, C. Ojeda-Aristizabal, and A. Drieschova for useful discussions. Support was provided by Conacyt and NSF DMR-1206513, and Computer resources by NERSC under Contract No. DE-AC02-05CH11231.

\section{Appendix A: Numerical details}

We use density functional theory (DFT) as implemented in the Quantum-ESPRESSO ${ }^{26}$ computer package with the generalized gradient approximation ${ }^{41}$ to the exchange-correlation energy functional. Convergence of the energy in the self-consistent iterations was verified to better than $10^{-8} \mathrm{Ry}$ and we used a $100 \mathrm{Ry}$ plane wave kinetic energy cutoff. A k-point mesh of $9 \times 9 \times 1$ $(9 \times 9 \times 9)$ was used in slab (bulk) calculations. We performed a fully-relativistic calculation with relativistic effects included in the pseudopotentials.

We first fully relax the lattice constants and the internal coordinates in bulk compounds. For $\mathrm{Bi}_{2} \mathrm{Se}_{3}$ we obtained the DFT optimized parameters $a=4.080 \AA$, $c=28.198 \AA$ and for $\mathrm{Bi}_{2} \mathrm{Te}_{2}$ Se $a=4.247 \AA, c=29.632 \AA$. Following a bulk structural relaxation we performed the relaxation of the internal coordinates in the slab geometry. We checked that the LDOS results did not change if we used $6 \mathrm{QL}$ instead of $9 \mathrm{QL}$ or if we used $20 \AA$ vacuum instead of $10 \AA$. We then constructed maximally localized Wannier functions ${ }^{33}$ and performed the Wannier interpolation $^{28}$ using the Wannier $90^{27}$ computer package. For initial projections we used $s$ and $p$-like atom-centered orbitals for both $\mathrm{Bi}$ and $\mathrm{Se}(\mathrm{Te})$ atoms.

In the non-thermal calculation discussed in section III B, the occupations of the lowest $N$ bands at each kpoint are fixed to one, where $N$ is the total pseudocharge of our unit-cell $(N=252)$. Filling up the lowest $N$ bands in a topological insulator corresponds to occupying states up to the Dirac point. 
1 W. Mönch, Semiconductor Surfaces and Interfaces (Springer, 2010).

${ }^{2}$ L. Kronik and Y. Shapira, Surf. Sci. Rep. 37, 1 (1999).

3 R. Roy, Phys. Rev. B 79, 195322 (2009).

4 J. E. Moore and L. Balents, Phys. Rev. B 75, 121306 (2007).

5 L. Fu and C. L. Kane, Phys. Rev. B 76, 045302 (2007).

6 M. Dzero, K. Sun, V. Galitski, and P. Coleman, Phys. Rev. Lett. 104, 106408 (2010).

7 X. Zhang, N. P. Butch, P. Syers, S. Ziemak, R. L. Greene, and J. Paglione, Phys. Rev. X 3, 011011 (2013).

8 M. Z. Hasan and J. E. Moore, Annu. Rev. Condens. Matter Phys. 2, 55 (2011).

9 T. Yokoyama and S. Murakami, Physica E: Lowdimensional Systems and Nanostructures 55, 1 (2014).

10 D. Hsieh, Y. Xia, D. Qian, L. Wray, J. H. Dil, F. Meier, J. Osterwalder, L. Patthey, J. G. Checkelsky, N. P. Ong, A. V. Fedorov, H. Lin, A. Bansil, D. Grauer, Y. S. Hor, R. J. Cava, and M. Z. Hasan, Nature 460, 1101 (2009).

11 M. Bianchi, D. Guan, S. Bao, J. Mi, B. B. Iversen, P. D. King, and P. Hofmann, Nat Commun 1, 128 (2010).

12 J. G. Analytis, J.-H. Chu, Y. Chen, F. Corredor, R. D. McDonald, Z. X. Shen, and I. R. Fisher, Phys. Rev. B 81, 205407 (2010).

13 C. Chen, S. He, H. Weng, W. Zhang, L. Zhao, H. Liu, X. Jia, D. Mou, S. Liu, J. He, Y. Peng, Y. Feng, Z. Xie, G. Liu, X. Dong, J. Zhang, X. Wang, Q. Peng, Z. Wang, S. Zhang, F. Yang, C. Chen, Z. Xu, X. Dai, Z. Fang, and X. J. Zhou, Proc. Natl. Acad. Sci. USA 109, 3694 (1012).

14 M. Neupane, S.-Y. Xu, L. A. Wray, A. Petersen, R. Shankar, N. Alidoust, C. Liu, A. Fedorov, H. Ji, J. M. Allred, Y. S. Hor, T.-R. Chang, H.-T. Jeng, H. Lin, A. Bansil, R. J. Cava, and M. Z. Hasan, Phys. Rev. 85, 235406 (2012).

15 M. T. Edmonds, J. T. Hellerstedt, A. Tadich, A. Schenk, K. M. ODonnell, J. Tosado, N. P. Butch, P. Syers, J. Paglione, and M. S. Fuhrer, The Journal of Physical Chemistry C 118, 20413 (2014).

16 E. Frantzeskakis, N. de Jong, B. Zwartsenberg, T. V. Bay, Y. K. Huang, S. V. Ramankutty, A. Tytarenko, D. Wu, Y. Pan, S. Hollanders, M. Radovic, N. C. Plumb, N. Xu, M. Shi, C. Lupulescu, T. Arion, R. Ovsyannikov, A. Varykhalov, W. Eberhardt, A. de Visser, E. van Heumen, and M. S. Golden, Phys. Rev. B 91, 205134 (2015).

17 S. Urazhdin, D. Bilc, S. D. Mahanti, S. H. Tessmer, T. Kyratsi, and M. G. Kanatzidis, Phys. Rev. B 69, 085313 (2004).

18 M. T. Pettes, J. Maassen, I. Jo, M. S. Lundstrom, and L. Shi, Nano Letters 13, 5316 (2013).

19 A. Narayan, I. Rungger, A. Droghetti, and S. Sanvito, Phys. Rev. B 90, 205431 (2014).

20 C. E. ViolBarbosa, C. Shekhar, B. Yan, S. Ouardi, E. Ikenaga, G. H. Fecher, and C. Felser, Phys. Rev. B 88, 195128 (2013).
21 P. D. C. King, T. D. Veal, and C. F. McConville, Phys. Rev. B 77, 125305 (2008).

22 M. Brahlek, N. Koirala, N. Bansal, and S. Oh, Solid State Communications 215, 54 (2015).

23 D. Galanakis and T. D. Stanescu, Phys. Rev. B 86, 195311 (2012).

24 M. Bahramy, P. King, A. de la Torre, J. Chang, M. Shi, L. Patthey, G. Balakrishnan, P. Hofmann, R. Arita, N. Nagaosa, and F. Baumberger, Nat Commun 3, 1159 (2012).

25 R. J. Cava, H. Ji, M. K. Fuccillo, Q. D. Gibson, and Y. S. Horb, J. Mater. Chem. C 1, 3176 (2013).

${ }^{26}$ P. Giannozzi et al., Journal of Physics: Condensed Matter 21, 395502 (2009).

27 A. A. Mostofi, J. R. Yates, Y.-S. Lee, I. Souza, D. Vanderbilt, and N. Marzari, Comput. Phys. Commun. 178, 685 (2008).

28 J. Yates, X. Wang, D. Vanderbilt, and I. Souza, Phys. Rev. B 75, 195121 (2007).

29 J. Black, E. Conwell, L. Seigle, and C. Spencer, Journal of Physics and Chemistry of Solids 2, 240 (1957).

30 Y. Xia, D. Qian, D. Hsieh, L. Wray, A. Pal, H. Lin, A. Bansil, D. Grauer, Y. S. Hor, R. J. Cava, and M. Z. Hasan, Nat. Phys. 5, 398 (2009).

31 S.-Y. Xu, L. A. Wray, Y. Xia, R. Shankar, A. Petersen, A. Fedorov, H. Lin, A. Bansil, Y. S. Hor, D. Grauer, R. J. Cava, and M. Z. Hasan, "Discovery of several large families of topological insulator classes with backscatteringsuppressed spin-polarized single-dirac-cone on the surface," ArXiv:1007.5111 [cond-mat.mes-hall].

32 D. Lovett, Semimetals and Narrow-bandgap Semiconductors (Pion Limited, London, UK, 1977).

${ }^{33}$ N. Marzari, A. A. Mostofi, J. R. Yates, I. Souza, and D. Vanderbilt, Rev. Mod. Phys. 84, 1419 (2012).

${ }^{34}$ O. Madelung, U. Rssler, and M. Schulz, eds., NonTetrahedrally Bonded Elements and Binary Compounds I (Springer, Berlin, 1998).

35 Z. Ren, A. A. Taskin, S. Sasaki, K. Segawa, and Y. Ando, Phys. Rev. B 82, 241306 (2010).

${ }^{36}$ K. Yang, W. Setyawan, S. Wang, M. Buongiorno Nardelli, and S. Curtarolo, Nat Mater 11, 614 (2012).

37 A. Marcinkova, J. K. Wang, C. Slavonic, A. H. Nevidomskyy, K. F. Kelly, Y. Filinchuk, and E. Morosan, Phys. Rev. B 88, 165128 (2013).

38 M. Arita, H. Sato, K. Shimada, H. Namatame, M. Taniguchi, M. Sasaki, M. Kitaura, A. Ohnishi, and H.-J. Kim, JPS Conf. Proc. 1, 012017 (2014).

39 K. Hoefera, C. Beckera, D. Rata, J. Swanson, P. Thalmeiera, and L. H. Tjenga, Proc. Natl. Acad. Sci. USA 111, 14979 (2014).

40 P. Rakyta, B. Ujfalussy, and L. Szunyogh, "Band bending at the surface of $\mathrm{bi}_{2} \mathrm{se}_{3}$ studied from first principles," ArXiv:1504.05071 [cond-mat.mes-hall].

41 J. P. Perdew, K. Burke, and M. Ernzerhof, Phys. Rev. Lett. 78, 1396 (1997). 